\title{
On a Max-Type Difference System
}

\section{Decun Zhang, Xibao Li, Liying Wang, Shiwei Cui}

Institute of Systems Science and Mathematics, Naval Aeronautical and Astronautical University, Yantai, China

Email: dczhang1967@tom.com, ytliyingwang@163.com

Received 22 August 2014; revised 25 September 2014; accepted 6 October 2014

Copyright (C) 2014 by authors and Scientific Research Publishing Inc.

This work is licensed under the Creative Commons Attribution International License (CC BY).

http://creativecommons.org/licenses/by/4.0/

c) (i) Open Access

\section{Abstract}

In this paper, we show that every well-defined solution of the max-type system of difference equations $x_{n+1}=\max \left\{\frac{\beta}{x_{n}}, y_{n-1}\right\}, y_{n+1}=\max \left\{\frac{\beta}{y_{n}}, x_{n+1}\right\}, n \in \mathbb{N}_{0}$ is eventually periodic with period four.

\section{Keywords}

Periodic Solution, Max-Type Difference System

\section{Introduction}

Max-type difference equations and max-type difference systems have been wisely applied in biology, computer science and automatic control systems and so on. There has been great interest in studying these equations in recent years.

For example, Briden et al. [1] investigated the periodicity character of the solution of the max-type difference equation

$$
x_{n+1}=\max \left\{\frac{1}{x_{n}}, \frac{A_{n}}{x_{n-1}}\right\}, \quad n \in \mathbb{N}_{0}
$$

Xiao Qian et al. [2] showed that the solution of the max-type difference equation

$$
x_{n+1}=\max \left\{\frac{\beta}{x_{n}}, x_{n-1}\right\}, \quad n \in \mathbb{N}_{0}
$$

is periodic with period two.

W. Q. Ji et al. [3] showed that the solution of the max-type difference system 


$$
\left\{\begin{array}{l}
x_{n+1}=\max \left\{\frac{\beta}{y_{n}}, x_{n-1}\right\} \\
y_{n+1}=\max \left\{\frac{\beta}{x_{n}}, y_{n-1}\right\}
\end{array}, \quad n \in \mathbb{N}_{0}\right.
$$

is periodic with period two.

In addition, E. M. Elasyed, Stevo Stević and others investigated some periodic max-type difference equations and periodic max-type difference systems in [4]-[7].

In this paper we show that every solution of the following max-type difference system

$$
\left\{\begin{array}{l}
x_{n+1}=\max \left\{\frac{\beta}{x_{n}}, y_{n-1}\right\} \\
y_{n+1}=\max \left\{\frac{\beta}{y_{n}}, x_{n-1}\right\}
\end{array}, \quad n=0,1, \cdots\right.
$$

where the initial conditions $x_{-1}, x_{0}, y_{-1}, y_{0}$ are arbitrary non-zero real numbers and $n \in \mathbb{R}$, is periodic with period four.

Remark 1. Note that if $\beta=0$, then System (1) becomes $x_{n+1}=y_{n-1}, x_{n-1}=y_{n+1}$, from which it follows that $x_{n}=x_{n+4}, y_{n}=y_{n+4}$ and every solution is periodic with period four.

\section{Some Lemmas}

Lemma 1 Assume that $\left\{x_{n}, y_{n}\right\}_{n=-1}^{\infty}$ is a solution of System (1) and there exists a $k_{0} \in\{-1,0,1,2, \cdots\}$ such that

$$
x_{k_{0}}=y_{k_{0}+2}, \quad x_{k_{0}+1}=y_{k_{0}+3}, \quad y_{k_{0}}=x_{k_{0}+2}, \quad y_{k_{0}+1}=x_{k_{0}+3}
$$

Then every solution is periodic with period four.

Proof Frist, we will prove that

$$
\begin{aligned}
& x_{k_{0}}=x_{k_{0}+4 m}, \quad x_{k_{0}+1}=x_{k_{0}+4 m+1}, \quad x_{k_{0}+2}=x_{k_{0}+4 m+2}, \quad x_{k_{0}+3}=x_{k_{0}+4 m+3} ; \\
& y_{k_{0}}=y_{k_{0}+4 m}, y_{k_{0}+1}=y_{k_{0}+4 m+1}, \quad y_{k_{0}+2}=y_{k_{0}+4 m+2}, \quad y_{k_{0}+3}=y_{k_{0}+4 m+3} \text {. }
\end{aligned}
$$

where $m \in \mathbb{N}$, from which the lemma follows.

Now, we use the method of induction. For $m=1$, Equation (3) becomes the following equations

$$
\begin{aligned}
& x_{k_{0}}=x_{k_{0}+4}, \quad x_{k_{0}+1}=x_{k_{0}+5}, \quad x_{k_{0}+2}=x_{k_{0}+6}, \quad x_{k_{0}+3}=x_{k_{0}+7} ; \\
& y_{k_{0}}=y_{k_{0}+4}, \quad y_{k_{0}+1}=y_{k_{0}+5}, \quad y_{k_{0}+2}=y_{k_{0}+6}, \quad y_{k_{0}+3}=y_{k_{0}+7} \text {. }
\end{aligned}
$$

By System (1) and Equation (2), we obtain that

$$
\begin{aligned}
& \left\{\begin{array}{l}
x_{k_{0}+4}=\max \left\{\frac{\beta}{x_{k_{0}+3}}, y_{k_{0}+2}\right\}=\max \left\{\frac{\beta}{y_{k_{0}+1}}, x_{k_{0}}\right\}=y_{k_{0}+2}=x_{k_{0}}, \\
y_{k_{0}+4}=\max \left\{\frac{\beta}{y_{k_{0}+3}}, x_{k_{0}+2}\right\}=\max \left\{\frac{\beta}{x_{k_{0}+1}}, y_{k_{0}}\right\}=x_{k_{0}+2}=y_{k_{0}},
\end{array}\right. \\
& \left\{\begin{array}{l}
x_{k_{0}+5}=\max \left\{\frac{\beta}{x_{k_{0}+4}}, y_{k_{0}+3}\right\}=\max \left\{\frac{\beta}{y_{k_{0}+2}}, x_{k_{0}+1}\right\}=y_{k_{0}+3}=x_{k_{0}+1}, \\
y_{k_{0}+5}=\max \left\{\frac{\beta}{y_{k_{0}+4}}, x_{k_{0}+3}\right\}=\max \left\{\frac{\beta}{x_{k_{0}+2}}, y_{k_{0}+1}\right\}=x_{k_{0}+3}=y_{k_{0}+1},
\end{array}\right.
\end{aligned}
$$




$$
\begin{aligned}
& \left\{\begin{array}{l}
x_{k_{0}+6}=\max \left\{\frac{\beta}{x_{k_{0}+5}}, y_{k_{0}+4}\right\}=\max \left\{\frac{\beta}{x_{k_{0}+1}}, y_{k_{0}}\right\}=x_{k_{0}+2}, \\
y_{k_{0}+6}=\max \left\{\frac{\beta}{y_{k_{0}+5}}, x_{k_{0}+4}\right\}=\max \left\{\frac{\beta}{y_{k_{0}+1}}, x_{k_{0}}\right\}=y_{k_{0}+2},
\end{array}\right. \\
& \left\{\begin{array}{l}
x_{k_{0}+7}=\max \left\{\frac{\beta}{x_{k_{0}+6}}, y_{k_{0}+5}\right\}=\max \left\{\frac{\beta}{x_{k_{0}+2}}, y_{k_{0}+1}\right\}=x_{k_{0}+3}, \\
y_{k_{0}+7}=\max \left\{\frac{\beta}{y_{k_{0}+6}}, x_{k_{0}+5}\right\}=\max \left\{\frac{\beta}{y_{k_{0}+2}}, x_{k_{0}+1}\right\}=y_{k_{0}+3} .
\end{array}\right.
\end{aligned}
$$

From which, Equation (4) holds.

Assume Equation (3) holds for $1 \leq m \leq m_{0}$, and by using System (1) and Equation (2), we obtain that

$$
\begin{aligned}
& \left\{\begin{array}{l}
x_{k_{0}+4\left(m_{0}+1\right)}=\max \left\{\frac{\beta}{x_{k_{0}+4 m_{0}+3}}, y_{k_{0}+4 m_{0}+2}\right\}=\max \left\{\frac{\beta}{x_{k_{0}+3}}, y_{k_{0}+2}\right\}=x_{k_{0}+4}=x_{k_{0}}, \\
y_{k_{0}+4\left(m_{0}+1\right)}=\max \left\{\frac{\beta}{y_{k_{0}+4 m_{0}+3}}, x_{k_{0}+4 m_{0}+2}\right\}=\max \left\{\frac{\beta}{y_{k_{0}+3}}, x_{k_{0}+2}\right\}=y_{k_{0}+4}=y_{k_{0}},
\end{array}\right. \\
& \left\{\begin{array}{l}
x_{k_{0}+1+4\left(m_{0}+1\right)}=\max \left\{\frac{\beta}{x_{k_{0}+4 m_{0}+4}}, y_{k_{0}+4 m_{0}+3}\right\}=\max \left\{\frac{\beta}{x_{k_{0}+4}}, y_{k_{0}+3}\right\}=x_{k_{0}+5}=x_{k_{0}+1}, \\
y_{k_{0}+1+4\left(m_{0}+1\right)}=\max \left\{\frac{\beta}{y_{k_{0}+4 m_{0}+4}}, x_{k_{0}+4 m_{0}+3}\right\}=\max \left\{\frac{\beta}{y_{k_{0}+4}}, x_{k_{0}+3}\right\}=y_{k_{0}+5}=y_{k_{0}+1},
\end{array}\right. \\
& \left\{\begin{array}{l}
x_{k_{0}+2+4\left(m_{0}+1\right)}=\max \left\{\frac{\beta}{x_{k_{0}+4 m_{0}+5}}, y_{k_{0}+4 m_{0}+4}\right\}=\max \left\{\frac{\beta}{x_{k_{0}+5}}, y_{k_{0}+4}\right\}=x_{k_{0}+6}=x_{k_{0}+2}, \\
y_{k_{0}+2+4\left(m_{0}+1\right)}=\max \left\{\frac{\beta}{y_{k_{0}+4 m_{0}+5}}, x_{k_{0}+4 m_{0}+4}\right\}=\max \left\{\frac{\beta}{y_{k_{0}+5}}, x_{k_{0}+4}\right\}=y_{k_{0}+6}=y_{k_{0}+2},
\end{array}\right. \\
& \left\{\begin{array}{l}
x_{k_{0}+3+4\left(m_{0}+1\right)}=\max \left\{\frac{\beta}{x_{k_{0}+4 m_{0}+6}}, y_{k_{0}+4 m_{0}+5}\right\}=\max \left\{\frac{\beta}{x_{k_{0}+6}}, y_{k_{0}+5}\right\}=x_{k_{0}+7}=x_{k_{0}+3}, \\
y_{k_{0}+3+4\left(m_{0}+1\right)}=\max \left\{\frac{\beta}{y_{k_{0}+4 m_{0}+6}}, x_{k_{0}+4 m_{0}+5}\right\}=\max \left\{\frac{\beta}{y_{k_{0}+6}}, x_{k_{0}+5}\right\}=y_{k_{0}+7}=y_{k_{0}+3} .
\end{array}\right.
\end{aligned}
$$

So we complete the proof.

Lemma 2 Assume that $\beta>0$. Then every solution of System (1) is positive if initial conditions satisfy one of the following conditions $x_{-1}>0$ or $x_{0}>0$ or $y_{-1}>0$ or $y_{0}>0$.

Proof Without loss of generality, we assume that $x_{-1}>0$ and from System (1) we have

$$
\begin{aligned}
& y_{1}=\max \left\{\frac{\beta}{y_{0}}, x_{-1}\right\}>0, \quad y_{2}=\max \left\{\frac{\beta}{y_{1}}, x_{0}\right\}>0 ; \\
& x_{3}=\max \left\{\frac{\beta}{x_{2}}, y_{1}\right\}>0, \quad x_{4}=\max \left\{\frac{\beta}{x_{3}}, y_{2}\right\}>0 .
\end{aligned}
$$

By using the method of induction, we have

$$
x_{n}, y_{n}>0, \quad n \geq 3
$$

Similarly, when $x_{0}>0$ or $y_{-1}>0$ or $y_{0}>0$, there exists an $n_{0} \in \mathbb{N}_{0}$ such that 


$$
x_{n}, y_{n}>0, \quad n \geq n_{0}
$$

The proof is completed.

Lemma 3 Assume that $\beta>0$. Then every solution of System (1) with positive initial conditions is periodic with period four.

Proof By System (1), we obtain that

$$
x_{1}=\max \left\{\frac{\beta}{x_{0}}, y_{-1}\right\}>0, \quad y_{1}=\max \left\{\frac{\beta}{y_{0}}, x_{-1}\right\}>0
$$

Let $p=\max \left\{y_{0}, x_{0}\right\}, q=\max \left\{\frac{\beta}{y_{-1}}, y_{0}\right\}, s=\max \left\{\frac{\beta}{x_{-1}}, x_{0}\right\}$ and there are four cases which need to be discussed.

Case 1. $\frac{\beta}{x_{0}} \geq y_{-1}, \frac{\beta}{y_{0}} \geq x_{-1}$. We have

$$
\begin{aligned}
& x_{1}=\max \left\{\frac{\beta}{x_{0}}, y_{-1}\right\}=\frac{\beta}{x_{0}}, \quad y_{1}=\max \left\{\frac{\beta}{y_{0}}, x_{-1}\right\}=\frac{\beta}{y_{0}} ; \\
& x_{2}=\max \left\{\frac{\beta}{x_{1}}, y_{0}\right\}=\max \left\{x_{0}, y_{0}\right\}=p, \quad y_{2}=\max \left\{\frac{\beta}{y_{1}}, x_{0}\right\}=\max \left\{y_{0}, x_{0}\right\}=p \\
& x_{3}=\max \left\{\frac{\beta}{x_{2}}, y_{1}\right\}=\max \left\{\frac{\beta}{p}, \frac{\beta}{y_{0}}\right\}=\frac{\beta}{y_{0}}=y_{1}, \quad y_{3}=\max \left\{\frac{\beta}{y_{2}}, x_{1}\right\}=\max \left\{\frac{\beta}{p}, \frac{\beta}{x_{0}}\right\}=\frac{\beta}{x_{0}}=x_{1} ; \\
& x_{4}=\max \left\{\frac{\beta}{x_{3}}, y_{2}\right\}=\max \left\{y_{0}, p\right\}=p=y_{2}, \quad y_{4}=\max \left\{\frac{\beta}{y_{3}}, x_{2}\right\}=\max \left\{x_{0}, p\right\}=p=x_{2} .
\end{aligned}
$$

Hence, $x_{1}=y_{3}, x_{2}=y_{4}, y_{1}=x_{3}, y_{2}=x_{4}$. And by Lemma 1 , we have that the solution is periodic with period four. Moreover, we have

$$
\begin{array}{ll}
x_{4 n+1}=y_{4 n+3}=x_{1}=\frac{\beta}{x_{0}}, & y_{4 n+1}=x_{4 n+3}=y_{1}=\frac{\beta}{y_{0}} ; \\
x_{4 n+2}=y_{4 n+4}=x_{2}=p, & y_{4 n+2}=x_{4 n+4}=y_{2}=p,
\end{array}
$$

where $n \in \mathbb{N}_{0}$, and the solution has the following form

$$
\begin{aligned}
& \left\{x_{n}\right\}_{n=-1}^{\infty}=\left\{x_{-1}, x_{0}, \frac{\beta}{x_{0}}, p, \frac{\beta}{y_{0}}, p, \frac{\beta}{x_{0}}, p, \frac{\beta}{y_{0}}, p, \cdots\right\}, \\
& \left\{y_{n}\right\}_{n=-1}^{\infty}=\left\{y_{-1}, y_{0}, \frac{\beta}{y_{0}}, p, \frac{\beta}{x_{0}}, p, \frac{\beta}{y_{0}}, p, \frac{\beta}{x_{0}}, p, \cdots\right\} .
\end{aligned}
$$

Case 2. $\frac{\beta}{x_{0}}<y_{-1}, \frac{\beta}{y_{0}} \geq x_{-1}$. We have

$$
\begin{aligned}
& x_{1}=\max \left\{\frac{\beta}{x_{0}}, y_{-1}\right\}=y_{-1}, \quad y_{1}=\max \left\{\frac{\beta}{y_{0}}, x_{-1}\right\}=\frac{\beta}{y_{0}} ; \\
& x_{2}=\max \left\{\frac{\beta}{x_{1}}, y_{0}\right\}=\max \left\{\frac{\beta}{y_{-1}}, y_{0}\right\}=q, \quad y_{2}=\max \left\{\frac{\beta}{y_{1}}, x_{0}\right\}=\max \left\{y_{0}, x_{0}\right\}=p \\
& x_{3}=\max \left\{\frac{\beta}{x_{2}}, y_{1}\right\}=\max \left\{\frac{\beta}{q}, \frac{\beta}{y_{0}}\right\}=\frac{\beta}{y_{0}}=y_{1}, \quad y_{3}=\max \left\{\frac{\beta}{y_{2}}, x_{1}\right\}=\max \left\{\frac{\beta}{p}, y_{-1}\right\}=y_{-1}=x_{1} ; \\
& x_{4}=\max \left\{\frac{\beta}{x_{3}}, y_{2}\right\}=\max \left\{y_{0}, p\right\}=p=y_{2}, \quad y_{4}=\max \left\{\frac{\beta}{y_{3}}, x_{2}\right\}=\max \left\{\frac{\beta}{y_{-1}}, q\right\}=q=x_{2} .
\end{aligned}
$$


Hence, $x_{1}=y_{3}, x_{2}=y_{4}, y_{1}=x_{3}, y_{2}=x_{4}$. And by Lemma 1 , we have that the solution is periodic with period four. Moreover, we have

$$
\begin{aligned}
& x_{4 n+1}=y_{4 n+3}=x_{1}=y_{-1}, \quad y_{4 n+1}=x_{4 n+3}=y_{1}=\frac{\beta}{y_{0}} ; \\
& x_{4 n+2}=y_{4 n+4}=x_{2}=q, \quad y_{4 n+2}=x_{4 n+4}=y_{2}=p,
\end{aligned}
$$

where $n \in \mathbb{N}_{0}$, and the solution has the following form

$$
\begin{aligned}
& \left\{x_{n}\right\}_{n=-1}^{\infty}=\left\{x_{-1}, x_{0}, y_{-1}, q, \frac{\beta}{y_{0}}, p, y_{-1}, q, \frac{\beta}{y_{0}}, p, \cdots\right\}, \\
& \left\{y_{n}\right\}_{n=-1}^{\infty}=\left\{y_{-1}, y_{0}, \frac{\beta}{y_{0}}, p, y_{-1}, q, \frac{\beta}{y_{0}}, p, y_{-1}, q, \cdots\right\} .
\end{aligned}
$$

Case 3. $\frac{\beta}{x_{0}} \geq y_{-1}, \frac{\beta}{y_{0}}<x_{-1}$. We have

$$
\begin{aligned}
& x_{1}=\max \left\{\frac{\beta}{x_{0}}, y_{-1}\right\}=\frac{\beta}{x_{0}}, \quad y_{1}=\max \left\{\frac{\beta}{y_{0}}, x_{-1}\right\}=x_{-1} ; \\
& x_{2}=\max \left\{\frac{\beta}{x_{1}}, y_{0}\right\}=\max \left\{x_{0}, y_{0}\right\}=p, \quad y_{2}=\max \left\{\frac{\beta}{y_{1}}, x_{0}\right\}=\max \left\{\frac{\beta}{x_{-1}}, x_{0}\right\}=s ; \\
& x_{3}=\max \left\{\frac{\beta}{x_{2}}, y_{1}\right\}=\max \left\{\frac{\beta}{p}, x_{-1}\right\}=x_{-1}=y_{1}, \quad y_{3}=\max \left\{\frac{\beta}{y_{2}}, x_{1}\right\}=\max \left\{\frac{\beta}{s}, \frac{\beta}{x_{0}}\right\}=\frac{\beta}{x_{0}}=x_{1} ; \\
& x_{4}=\max \left\{\frac{\beta}{x_{3}}, y_{2}\right\}=\max \left\{\frac{\beta}{x_{-1}}, s\right\}=s=y_{2}, \quad y_{4}=\max \left\{\frac{\beta}{y_{3}}, x_{2}\right\}=\max \left\{x_{0}, p\right\}=p=x_{2} .
\end{aligned}
$$

Hence, $x_{1}=y_{3}, x_{2}=y_{4}, y_{1}=x_{3}, y_{2}=x_{4}$. And by Lemma 1 , we have that the solution is periodic with period four. Moreover, we have

$$
\begin{array}{ll}
x_{4 n+1}=y_{4 n+3}=x_{1}=\frac{\beta}{x_{0}}, & y_{4 n+1}=x_{4 n+3}=y_{1}=x_{-1} ; \\
x_{4 n+2}=y_{4 n+4}=x_{2}=p, & y_{4 n+2}=x_{4 n+4}=y_{2}=s,
\end{array}
$$

where $n \in \mathbb{N}_{0}$, and the solution has the following form

$$
\begin{aligned}
& \left\{x_{n}\right\}_{n=-1}^{\infty}=\left\{x_{-1}, x_{0}, \frac{\beta}{x_{0}}, p, x_{-1}, s, \frac{\beta}{x_{0}}, p, x_{-1}, s, \cdots\right\}, \\
& \left\{y_{n}\right\}_{n=-1}^{\infty}=\left\{y_{-1}, y_{0}, x_{-1}, s, \frac{\beta}{x_{0}}, p, x_{-1}, s, \frac{\beta}{x_{0}}, p, \cdots\right\} .
\end{aligned}
$$

Case 4. $\frac{\beta}{x_{0}}<y_{-1}, \frac{\beta}{y_{0}}<x_{-1}$. We have

$$
\begin{aligned}
& x_{1}=\max \left\{\frac{\beta}{x_{0}}, y_{-1}\right\}=y_{-1}, \quad y_{1}=\max \left\{\frac{\beta}{y_{0}}, x_{-1}\right\}=x_{-1} ; \\
& x_{2}=\max \left\{\frac{\beta}{x_{1}}, y_{0}\right\}=\max \left\{\frac{\beta}{y_{-1}}, y_{0}\right\}=q, \quad y_{2}=\max \left\{\frac{\beta}{y_{1}}, x_{0}\right\}=\max \left\{\frac{\beta}{x_{-1}}, x_{0}\right\}=s ; \\
& x_{3}=\max \left\{\frac{\beta}{x_{2}}, y_{1}\right\}=\max \left\{\frac{\beta}{q}, x_{-1}\right\}=x_{-1}=y_{1}, \quad y_{3}=\max \left\{\frac{\beta}{y_{2}}, x_{1}\right\}=\max \left\{\frac{\beta}{s}, y_{-1}\right\}=y_{-1}=x_{1} ; \\
& x_{4}=\max \left\{\frac{\beta}{x_{3}}, y_{2}\right\}=\max \left\{\frac{\beta}{x_{-1}}, s\right\}=s=y_{2}, \quad y_{4}=\max \left\{\frac{\beta}{y_{3}}, x_{2}\right\}=\max \left\{\frac{\beta}{y_{-1}}, q\right\}=q=x_{2} .
\end{aligned}
$$


Hence, $x_{1}=y_{3}, x_{2}=y_{4}, y_{1}=x_{3}, y_{2}=x_{4}$. And by Lemma 1, we have that the solution is periodic with period four. Moreover, we have

$$
\begin{aligned}
& x_{4 n+1}=y_{4 n+3}=x_{1}=y_{-1}, \quad y_{4 n+1}=x_{4 n+3}=y_{1}=x_{-1} \text {; } \\
& x_{4 n+2}=y_{4 n+4}=x_{2}=q, \quad y_{4 n+2}=x_{4 n+4}=y_{2}=s,
\end{aligned}
$$

where $n \in \mathbb{N}_{0}$, and the solution has the following form

$$
\begin{aligned}
& \left\{x_{n}\right\}_{n=-1}^{\infty}=\left\{x_{-1}, x_{0}, y_{-1}, q, x_{-1}, s, y_{-1}, q, x_{-1}, s, \cdots\right\}, \\
& \left\{y_{n}\right\}_{n=-1}^{\infty}=\left\{y_{-1}, y_{0}, x_{-1}, s, y_{-1}, q, x_{-1}, s, y_{-1}, q, \cdots\right\} .
\end{aligned}
$$

So we complete the proof.

Lemma 4 Assume that $\beta>0$. Then every solution of System (1) with negative initial conditions is periodic with period four.

Proof Since $x_{-1}, x_{0}, y_{-1}, y_{0}<0$ and $\beta>0$, by induction we have $x_{n}<0, y_{n}<0$. If we use the change $z_{n}=-x_{n}, w_{n}=-y_{n}$ and System (1) can be rewritten as follows

$$
\left\{\begin{array}{l}
z_{n+1}=\min \left\{\frac{\beta}{z_{n}}, w_{n-1}\right\}, \\
w_{n+1}=\min \left\{\frac{\beta}{w_{n}}, z_{n-1}\right\} .
\end{array}\right.
$$

where $z_{n}, w_{n}>0, n=-1,0,1, \cdots$.

Now, we will prove that every solution of System (5) with positive initial conditions is periodic with period four.

Let $\quad p^{\prime}=\min \left\{z_{0}, w_{0}\right\}, \quad q^{\prime}=\min \left\{\frac{\beta}{w_{-1}}, w_{0}\right\}, s^{\prime}=\min \left\{\frac{\beta}{z_{-1}}, z_{0}\right\}$. Similar to the proof of Lemma (3), there are four cases which need to be discussed.

Case 1. $\frac{\beta}{z_{0}}<w_{-1}, \frac{\beta}{w_{0}}<z_{-1}$. We obtain that

$$
\begin{aligned}
& \left\{z_{n}\right\}_{n=-1}^{\infty}=\left\{z_{-1}, z_{0}, \frac{\beta}{z_{0}}, p^{\prime}, \frac{\beta}{w_{0}}, p^{\prime}, \frac{\beta}{z_{0}}, p^{\prime}, \frac{\beta}{w_{0}}, p^{\prime}, \cdots\right\}, \\
& \left\{w_{n}\right\}_{n=-1}^{\infty}=\left\{w_{-1}, w_{0}, \frac{\beta}{w_{0}}, p^{\prime}, \frac{\beta}{z_{0}}, p^{\prime}, \frac{\beta}{w_{0}}, p^{\prime}, \frac{\beta}{z_{0}}, p^{\prime}, \cdots\right\} .
\end{aligned}
$$

Case 2. $\frac{\beta}{z_{0}}>w_{-1}, \frac{\beta}{w_{0}}<z_{-1}$. We obtain that

$$
\begin{aligned}
& \left\{z_{n}\right\}_{n=-1}^{\infty}=\left\{z_{-1}, z_{0}, w_{-1}, q^{\prime}, \frac{\beta}{w_{0}}, p^{\prime}, w_{-1}, q^{\prime}, \frac{\beta}{w_{0}}, p^{\prime}, \cdots\right\}, \\
& \left\{w_{n}\right\}_{n=-1}^{\infty}=\left\{w_{-1}, w_{0}, \frac{\beta}{w_{0}}, p^{\prime}, w_{-1}, q^{\prime}, \frac{\beta}{w_{0}}, p^{\prime}, w_{-1}, q^{\prime}, \cdots\right\} .
\end{aligned}
$$

Case 3. $\frac{\beta}{z_{0}}<w_{-1}, \frac{\beta}{w_{0}}>z_{-1}$. We obtain that

$$
\begin{aligned}
& \left\{z_{n}\right\}_{n=-1}^{\infty}=\left\{z_{-1}, z_{0}, \frac{\beta}{z_{0}}, p^{\prime}, z_{-1}, s^{\prime}, \frac{\beta}{z_{0}}, p^{\prime}, z_{-1}, s^{\prime}, \cdots\right\}, \\
& \left\{w_{n}\right\}_{n=-1}^{\infty}=\left\{w_{-1}, w_{0}, z_{-1}, s^{\prime}, \frac{\beta}{z_{0}}, p^{\prime}, z_{-1}, s^{\prime}, \frac{\beta}{z_{0}}, p^{\prime}, \cdots\right\} .
\end{aligned}
$$


Case 4. $\frac{\beta}{z_{0}}>w_{-1}, \frac{\beta}{w_{0}}>z_{-1}$. We obtain that

$$
\begin{aligned}
& \left\{z_{n}\right\}_{n=-1}^{\infty}=\left\{z_{-1}, z_{0}, w_{-1}, q^{\prime}, z_{-1}, s^{\prime}, w_{-1}, q^{\prime}, z_{-1}, s^{\prime}, \cdots\right\}, \\
& \left\{w_{n}\right\}_{n=-1}^{\infty}=\left\{w_{-1}, w_{0}, z_{-1}, s^{\prime}, w_{-1}, q^{\prime}, z_{-1}, s^{\prime}, w_{-1}, q^{\prime}, \cdots\right\} .
\end{aligned}
$$

So we complete the proof.

Lemma 5 Assume that $\beta<0$. Then every solution of System (1) is periodic with period four if initial conditions satisfy one of the following conditions

$$
\begin{aligned}
& \text { (a) } x_{-1}, x_{0}, y_{-1}, y_{0}>0 ; \quad \text { (b) } x_{-1}, x_{0}, y_{-1}, y_{0}<0 ; \\
& \text { (c) } x_{-1}, y_{0}>0, x_{0}, y_{-1}<0 ; \quad \text { (d) } x_{-1}, y_{0}<0, x_{0}, y_{-1}>0 \text {. }
\end{aligned}
$$

Proof (a) If $x_{-1}, x_{0}, y_{-1}, y_{0}>0$, by using System (1), we know that there is only one case which needs to be discussed. That is

$$
\frac{\beta}{x_{0}}<y_{-1}, \quad \frac{\beta}{y_{0}}<x_{-1}
$$

Then we have

$$
\begin{aligned}
& x_{1}=\max \left\{\frac{\beta}{x_{0}}, y_{-1}\right\}=y_{-1}>0, \quad y_{1}=\max \left\{\frac{\beta}{y_{0}}, x_{-1}\right\}=x_{-1}>0 ; \\
& x_{2}=\max \left\{\frac{\beta}{x_{1}}, y_{0}\right\}=y_{0}>0, \quad y_{2}=\max \left\{\frac{\beta}{y_{1}}, x_{0}\right\}=x_{0}>0 ; \\
& x_{3}=\max \left\{\frac{\beta}{x_{2}}, y_{1}\right\}=y_{1}=x_{-1}>0, \quad y_{3}=\max \left\{\frac{\beta}{y_{2}}, x_{1}\right\}=x_{1}=y_{-1}>0 ; \\
& x_{4}=\max \left\{\frac{\beta}{x_{3}}, y_{2}\right\}=y_{2}=x_{0}>0, \quad y_{4}=\max \left\{\frac{\beta}{y_{3}}, x_{2}\right\}=x_{2}=y_{0}>0 .
\end{aligned}
$$

Hence, $x_{1}=y_{3}, x_{2}=y_{4}, y_{1}=x_{3}, y_{2}=x_{4}$. And by Lemma 1 , we have that the solution is periodic with period four.

The proof of case $(b)(c)(d)$ is similar to the proof of case $(a)$, so we omit it. Then, the proof is completed.

Lemma 6 Assume that $\beta<0$. Then every solution of System (1) is periodic with period four if initial conditions satisfy one of the following conditions

$$
\begin{array}{ll}
\text { (e) } x_{-1}<0, x_{0}, y_{-1}, y_{0}>0 ; & \text { (f) } x_{0}<0, x_{-1}, y_{-1}, y_{0}>0 \\
\text { (j) } y_{-1}<0, x_{-1}, x_{0}, y_{0}>0 ; & \text { (h) } y_{0}<0, x_{-1}, x_{0}, y_{-1}>0 \\
\text { (i) } x_{-1}>0, x_{0}, y_{-1}, y_{0}<0 ; & \text { (j) } x_{0}>0, x_{-1}, y_{-1}, y_{0}<0 \\
\text { (k) } y_{-1}<0, x_{-1}, x_{0}, y_{0}>0 ; & \text { (l) } y_{0}>0, x_{-1}, x_{0}, y_{-1}<0
\end{array}
$$

Proof $(e)$ If $x_{-1}<0, x_{0}, y_{-1}, y_{0}>0$, by using System (1), we know that $\frac{\beta}{x_{0}}<y_{-1}$ so there are two cases which need to be discussed. That is

$$
\text { (e1) } \frac{\beta}{y_{0}} \geq x_{-1}, \quad(e 2) \frac{\beta}{y_{0}}<x_{-1}
$$

Case 1. (e1) $\frac{\beta}{y_{0}} \geq x_{-1}$. We have 


$$
\begin{aligned}
& x_{1}=\max \left\{\frac{\beta}{x_{0}}, y_{-1}\right\}=y_{-1}>0, \quad y_{1}=\max \left\{\frac{\beta}{y_{0}}, x_{-1}\right\}=\frac{\beta}{y_{0}}<0 \\
& x_{2}=\max \left\{\frac{\beta}{x_{1}}, y_{0}\right\}=\max \left\{\frac{\beta}{y_{-1}}, y_{0}\right\}=y_{0}>0, \quad y_{2}=\max \left\{\frac{\beta}{y_{1}}, x_{0}\right\}=\max \left\{y_{0}, x_{0}\right\}=p>0 \\
& x_{3}=\max \left\{\frac{\beta}{x_{2}}, y_{1}\right\}=\max \left\{\frac{\beta}{y_{0}}, \frac{\beta}{y_{0}}\right\}=\frac{\beta}{y_{0}}=y_{1}<0, \quad y_{3}=\max \left\{\frac{\beta}{y_{2}}, x_{1}\right\}=\max \left\{\frac{\beta}{p}, y_{-1}\right\}=y_{-1}=x_{1}>0 \\
& x_{4}=\max \left\{\frac{\beta}{x_{3}}, y_{2}\right\}=\max \left\{y_{0}, p\right\}=p=y_{2}>0, \quad y_{4}=\max \left\{\frac{\beta}{y_{3}}, x_{2}\right\}=\max \left\{\frac{\beta}{y_{-1}}, y_{0}\right\}=y_{0}=x_{2}>0 .
\end{aligned}
$$

Hence, $x_{1}=y_{3}, x_{2}=y_{4}, y_{1}=x_{3}, y_{2}=x_{4}$. And by Lemma 1, we have that the solution is periodic with period four.

Case 2. (e2) $\frac{\beta}{y_{0}}<x_{-1}$. We have

$$
\begin{aligned}
& x_{1}=\max \left\{\frac{\beta}{x_{0}}, y_{-1}\right\}=y_{-1}>0, \quad y_{1}=\max \left\{\frac{\beta}{y_{0}}, x_{-1}\right\}=x_{-1}<0 \\
& x_{2}=\max \left\{\frac{\beta}{x_{1}}, y_{0}\right\}=\max \left\{\frac{\beta}{y_{-1}}, y_{0}\right\}=y_{0}>0, \quad y_{2}=\max \left\{\frac{\beta}{y_{1}}, x_{0}\right\}=\max \left\{\frac{\beta}{x_{-1}}, x_{0}\right\}=s>0 ; \\
& x_{3}=\max \left\{\frac{\beta}{x_{2}}, y_{1}\right\}=\max \left\{\frac{\beta}{y_{0}}, x_{-1}\right\}=x_{-1}=y_{1}<0, \quad y_{3}=\max \left\{\frac{\beta}{y_{2}}, x_{1}\right\}=\max \left\{\frac{\beta}{s}, y_{-1}\right\}=y_{-1}=x_{1}>0 ; \\
& x_{4}=\max \left\{\frac{\beta}{x_{3}}, y_{2}\right\}=\max \left\{\frac{\beta}{x_{-1}}, s\right\}=s=y_{2}>0, \quad y_{4}=\max \left\{\frac{\beta}{y_{3}}, x_{2}\right\}=\max \left\{\frac{\beta}{y_{-1}}, y_{0}\right\}=y_{0}=x_{2}>0 .
\end{aligned}
$$
four.

Hence, $x_{1}=y_{3}, x_{2}=y_{4}, y_{1}=x_{3}, y_{2}=x_{4}$. And by Lemma 1, we have that the solution is periodic with period

The proof of case $(f)-(l)$ is similar to the proof of case $(e)$, so we omit it. Then, the proof is completed.

Lemma 7 Assume that $\beta<0$. Then every solution of System (1) is periodic with period four if initial conditions satisfy one of the following conditions

$(m) x_{-1}, x_{0}>0, y_{-1}, y_{0}<0 ;(n) x_{-1}, x_{0}<0, y_{-1}, y_{0}>0 ;(0) x_{-1}, y_{-1}>0, x_{0}, y_{0}<0 ;(p) x_{-1}, y_{-1}<0, x_{0}, y_{0}>0$.

Proof $(m)$ If $x_{-1}, x_{0}>0, y_{-1}, y_{0}<0$, by using System (1), we know that there are four cases which need to be discussed. That is

(m1) $\frac{\beta}{x_{0}} \geq y_{-1}, \frac{\beta}{y_{0}} \geq x_{-1} ;(m 2) \frac{\beta}{x_{0}}<y_{-1}, \frac{\beta}{y_{0}} \geq x_{-1} ;(m 3) \frac{\beta}{x_{0}} \geq y_{-1}, \frac{\beta}{y_{0}}<x_{-1} ;(m 4) \frac{\beta}{x_{0}}<y_{-1}, \frac{\beta}{y_{0}}<x_{-1}$.

Case 1. (m1) $\frac{\beta}{x_{0}} \geq y_{-1}, \frac{\beta}{y_{0}} \geq x_{-1}$. We have

$$
\begin{aligned}
& x_{1}=\max \left\{\frac{\beta}{x_{0}}, y_{-1}\right\}=\frac{\beta}{x_{0}}<0, \quad y_{1}=\max \left\{\frac{\beta}{y_{0}}, x_{-1}\right\}=\frac{\beta}{y_{0}}>0 \\
& x_{2}=\max \left\{\frac{\beta}{x_{1}}, y_{0}\right\}=\max \left\{x_{0}, y_{0}\right\}=x_{0}>0, \quad y_{2}=\max \left\{\frac{\beta}{y_{1}}, x_{0}\right\}=\max \left\{y_{0}, x_{0}\right\}=x_{0}>0 \\
& x_{3}=\max \left\{\frac{\beta}{x_{2}}, y_{1}\right\}=\max \left\{\frac{\beta}{x_{0}}, \frac{\beta}{y_{0}}\right\}=\frac{\beta}{y_{0}}=y_{1}>0, \quad y_{3}=\max \left\{\frac{\beta}{y_{2}}, x_{1}\right\}=\max \left\{\frac{\beta}{x_{0}}, \frac{\beta}{x_{0}}\right\}=\frac{\beta}{x_{0}}=x_{1}<0 \\
& x_{4}=\max \left\{\frac{\beta}{x_{3}}, y_{2}\right\}=\max \left\{y_{0}, x_{0}\right\}=x_{0}=y_{2}>0, \quad y_{4}=\max \left\{\frac{\beta}{y_{3}}, x_{2}\right\}=\max \left\{x_{0}, x_{0}\right\}=x_{0}=x_{2}>0
\end{aligned}
$$


Hence, $x_{1}=y_{3}, x_{2}=y_{4}, y_{1}=x_{3}, y_{2}=x_{4}$. And by Lemma 1, we have that the solution is periodic with period four.

The proof of case $(m 2)(m 3)(m 4)$ is similar to the proof of case $(b)(c)(d)$ in Lemma 3, so we omit it and case $(m)$ is completed.

Similarly, the proof of case $(n)(o)(p)$ is similar to the proof of case $(m)$, so we omit it.

\section{Main Results}

By using Lemma 2 and Lemma 3, we obtain the following result.

Theorem 1 Assume that $\beta>0$. Then every solution of System (1) is periodic with period four if initial conditions satisfies one of the following conditions $x_{-1}>0$ or $x_{0}>0$ or $y_{-1}>0$ or $y_{0}>0$.

By using Theorem 1 and Lemma 4, we obtain the following result.

Theorem 2 Assume that $\beta>0$. Then every well-defined solution of System (1) is periodic with period four. By using Lemma 5, Lemma 6 and Lemma 7, we obtain the following result.

Theorem 3 Assume that $\beta<0$. Then every well-defined solution of System (1) is periodic with period four. By using Theorem 2 and Theorem 3, we obtain the following result.

Theorem 4 Assume that $\beta \in \mathfrak{R}$. Then every well-defined solution of System (1) is periodic with period four.

\section{Acknowledgements}

We thank the Editor and the referee for their comments. Research supported by Distinguished Expert Foundation and Youth Science Foundation of Naval Aeronautical and Astronautical University.

\section{References}

[1] Briden, W.J., Grove, E.A., Kent, C.M. and Ladas, G. (1999) Eventually Periodic Solutions of $x_{n+1}=\max \left\{\frac{1}{x_{n}}, \frac{A_{n}}{x_{n-1}}\right\}$, Nonlinear Analysis, 6, 31-34.

[2] Xiao, Q. and Shi, Q.H. (2013) Eventually Periodic Solutions of a Max-Type Equation. Mathematical and Computer Modelling, 57, 992-996. http://dx.doi.org/10.1016/j.mcm.2012.10.010

[3] Ji, W.Q. (2013) On the Behavior of the Solution of Several Difference Equations and the Difference System. Naval Aeronautical Engineering Institute, Yantai.

[4] Simsek, D., Cinar, C. and Yalcinkaya, I. (2006) On the Solutions of the Difference Equation $x_{n}=\max \left\{x_{n-1}, \frac{1}{x_{n-1}}\right\}$. International Journal of Contemporary Mathematical Sciences, 1, 481-487.

[5] Elsayed, E.M. and Stević, S. (2009) On the Max-Type Equation $x_{n}=\max \left\{\frac{A}{x_{n}}, x_{n-1}\right\}$. Nonlinear Analysis, 71, 910-922. http://dx.doi.org/10.1016/j.na.2008.11.016

[6] Stevć, S. (2012) On Some Periodic Systems of Max-Type Difference Equations. Applied Mathematics and Computation, 218, 11483-11487. http://dx.doi.org/10.1016/j.amc.2012.04.077

[7] Stević, S. (2012) Solutions of a Max-Type System of Difference Equations. Applied Mathematics and Computation, 218, 9825-9830. http://dx.doi.org/10.1016/j.amc.2012.03.057 
Scientific Research Publishing (SCIRP) is one of the largest Open Access journal publishers. It is currently publishing more than 200 open access, online, peer-reviewed journals covering a wide range of academic disciplines. SCIRP serves the worldwide academic communities and contributes to the progress and application of science with its publication.

Other selected journals from SCIRP are listed as below. Submit your manuscript to us via either submit@scirp.org or Online Submission Portal.
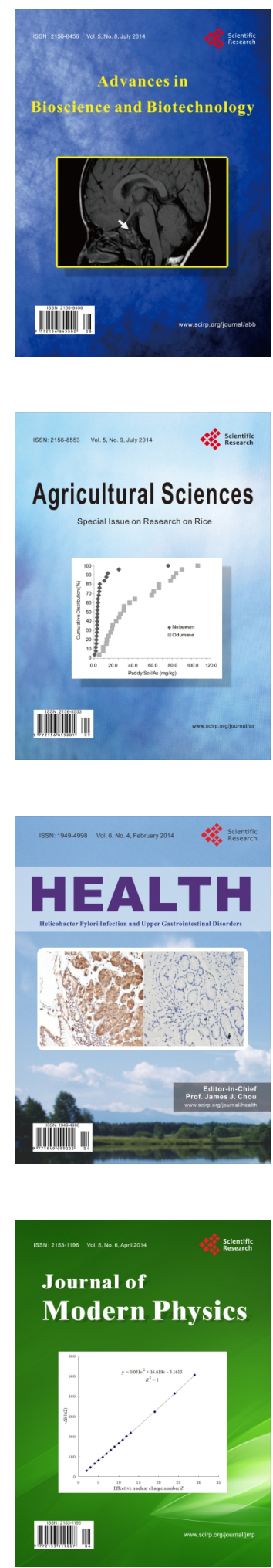
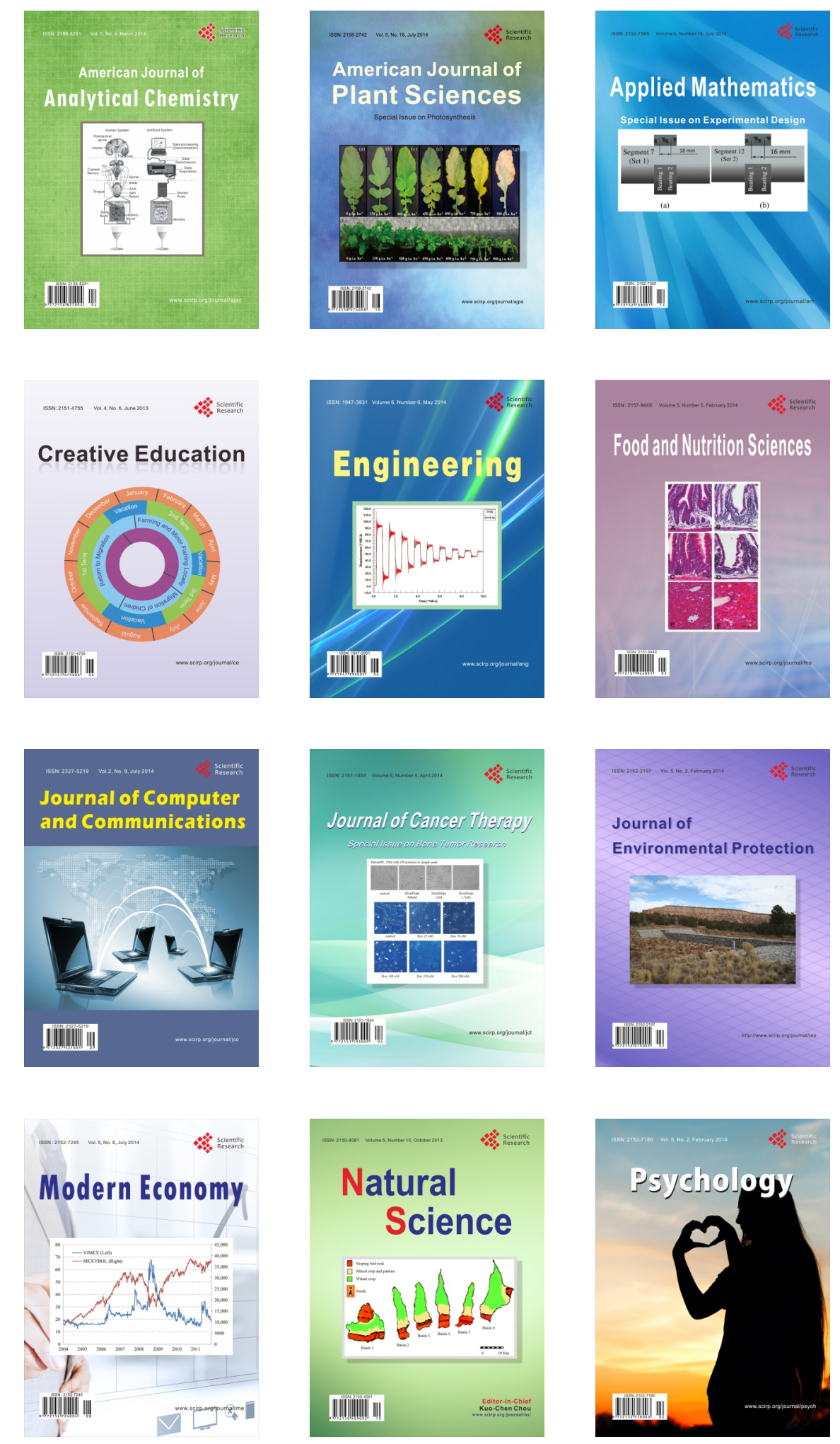